\title{
A new analytical framework of 'continuum of prevention and care' to maximize HIV case detection and retention in care in Vietnam
}

Masami Fujita ${ }^{1 *}$, Krishna C Poudel $^{2}$, Nhan Do Thi ${ }^{3}$, Duong Bui Duc ${ }^{3}$, Kinh Nguyen Van ${ }^{4}$, Kimberly Green ${ }^{5}$, Thu Nguyen Thi Minh ${ }^{6}$, Masaya Kato ${ }^{6}$, David Jacka ${ }^{6}$, Thuy Cao Thi Thanh ${ }^{4}$, Long Nguyen Thanh ${ }^{3}$ and Masamine Jimba ${ }^{7}$

\begin{abstract}
Background: The global initiative 'Treatment 2.0' calls for expanding the evidence base of optimal HIV service delivery models to maximize HIV case detection and retention in care. However limited systematic assessment has been conducted in countries with concentrated HIV epidemic. We aimed to assess HIV service availability and service connectedness in Vietnam.

Methods: We developed a new analytical framework of the continuum of prevention and care (COPC). Using the framework, we examined HIV service delivery in Vietnam. Specifically, we analyzed HIV service availability including geographical distribution and decentralization and service connectedness across multiple services and dimensions. We then identified system-related strengths and constraints in improving HIV case detection and retention in care. This was accomplished by reviewing related published and unpublished documents including existing service delivery data.

Results: Identified strengths included: decentralized HIV outpatient clinics that offer comprehensive care at the district level particularly in high HIV burden provinces; functional chronic care management for antiretroviral treatment (ART) with the involvement of people living with HIV and the links to community- and home-based care; HIV testing and counseling integrated into tuberculosis and antenatal care services in districts supported by donor-funded projects, and extensive peer outreach networks that reduce barriers for the most-at-risk populations to access services. Constraints included: fragmented local coordination mechanisms for HIV-related health services; lack of systems to monitor the expansion of HIV outpatient clinics that offer comprehensive care; underdevelopment of pre-ART care; insufficient linkage from HIV testing and counseling to pre-ART care; inadequate access to HIV-related services in districts not supported by donor-funded projects particularly in middle and low burden provinces and in mountainous remote areas; and no systematic monitoring of referral services.
\end{abstract}

Conclusions: Our COPC analytical framework was instrumental in identifying system-related strengths and constraints that contribute to HIV case detection and retention in care. The national HIV program plans to strengthen provincial programming by re-defining various service linkages and accelerate the transition from project-based approach to integrated service delivery in line with the 'Treatment 2.0 ' initiative.

Keywords: HIV, Health services, Health care systems, Developing countries

\footnotetext{
* Correspondence: fujitam@wpro.who.int

${ }^{1}$ World Health Organization Cambodia Office, P.O. Box 1217, No. 177-179

Pasteur (St.51) (corner 254) Sangkat Chak Tomouk, Phnom Penh, Cambodia

Full list of author information is available at the end of the article
} 


\section{Background}

Many resource-limited countries are facing significant challenges to delivering HIV health services. The first challenge is delayed HIV diagnosis and late presentation for antiretroviral treatment (ART) resulting in elevated morbidity and mortality [1] and in diminished benefit of ART as prevention [2]. The second one is a slow progress in expanding prevention of mother-to-child transmission (PMTCT) and tuberculosis (TB) - HIV collaborative activities [3]. Third, HIV care and support services are under-prioritized and under-funded, resulting in reduced access for pre-ART care, ART and end-of life patients [4]. Fourth, increasing number of PLHIV on ART for many years are facing long-term ART side effects, drug resistance, co-morbidities, and psychosocial constraints $[5,6]$. In addition to these challenges, the needs of most-at-risk populations (MARPs) have been overlooked in terms of HIV prevention, testing and counseling, and treatment particularly in Asia [7].

In these poor countries, project-based service delivery systems have been developed through urgent service expansion with massive donor funding over the last decade. In light of the current economic downturn, the new global initiative 'Treatment 2.0' highlights the needs to adapt delivery systems by decentralizing and integrating HIV treatment with other services and engaging community [8]. These services include drug dependence service, maternal and child health $(\mathrm{MCH})$ and tuberculosis (TB) services. The adaptation of delivery systems is expected to maximize HIV case detection and retention in care. The initiative calls for expanding the evidence base on optimal service delivery models in a variety of settings [9].

To date, limited systematic assessment has been conducted on overall models of HIV service delivery. Analyses of HIV service delivery have been fragmented. The focus has been on either one component of HIV health services such as ART [10], or integration between two services such as HIV and TB [11], HIV and $\mathrm{MCH}$ [12] or HIV and family planning [13]. The concept of the continuum of care, more recently understood as the continuum of prevention and care (COPC), has been used to coordinate and link the health facilities, the community and other sectors under one coherent framework [14-18]. We theorized that assessing service delivery models using the COPC concept could contribute to the optimization of HIV service delivery particularly to improving HIV case detection and retention in care in concentrated epidemic settings.

The first assessment using the COPC framework was conducted in Vietnam among other Asian countries. The HIV epidemic in Vietnam is largely concentrated among injecting drug users (IDUs), sex workers (SWs) and men who have sex with men (MSM). Adult HIV prevalence (aged 15-49) was estimated to be $0.44 \%$ with an estimated 254,000 PLHIV in 2010 [19]. HIV cases have been reported nationwide in all 63 provinces/cities, $98 \%$ of districts, and $74 \%$ of wards/communes (VAAC: Unpublished report; 2010).

This study aimed to assess HIV service delivery in Vietnam by identifying system related strengths and constraints that are common to multiple elements of HIV health services using the lens of the COPC. Specifically, we aimed to assess service availability including geographical distribution and decentralization and service connectedness across multiple services and dimensions that contribute to HIV case detection and retention in care. Vietnam has several relevant characteristics in common with many other Asian countries, such as low HIV prevalence in the general population but high prevalence among various MARPs [7], enormous variation of HIV prevalence across geographical areas, and structural barriers related to working with MARPs [20]. By providing a systematic analysis of the experience in Vietnam, we hope that such information will be useful in strengthening HIV service delivery of other resource-limited countries, particularly in Asia.

\section{Methods}

\section{Definition of COPC}

The COPC originates from the concept of the continuum of care developed in the 1970s to offer continuity of care for the elderly [21]. This concept emerged in the field of HIV/AIDS in 1990s when there were limited measures to prevent and treat HIV [22,23]. As the availability of HIV prevention, care and treatment has improved in resource limited settings, the concept has further evolved to include prevention [24,25].

The COPC can be defined as a coordinated network of prevention, treatment, care, and support activities. This network includes government, community-based organizations, non-government organizations, PLHIV and/or MARPs, their family members and others. This network spans different levels of the health system including the community. The resulting activities provide comprehensive services for adults, children, youth and families vulnerable to, living with and affected by HIV over the long-term [26] (Fujita M and Green K: Unpublished presentation; 2010).

A critical element of the COPC is to establish a comprehensive care site (CCS) as a central mechanism of a local service network. The CCS offers not only clinical care but a wide range of associated services. Such services include health education, psychosocial support, links to other services and community- and home-based care (CHBC), as well as opportunities for the involvement of affected communities such as MARPs and PLHIV. The names given to the CCS differ across Asia, such as the Day Care Centre, the Comprehensive Continuum of Care Centre, 
and the Friend-Help-Friend Centre [27]. Since HIV outpatient clinic is a common term used for HIV care and treatment facilities in Vietnam, in this paper, the term HIV outpatient 'plus' is used to denote activities and services of the CCS.

\section{Analytical framework}

We developed a new analytical framework of COPC for the review of HIV service delivery in Vietnam (Figure 1). The COPC concept can be applied to analyze HIV service delivery across several dimensions: (i) geographical distribution of MARPs and PLHIV; (ii) availability of HIV health services including their geographical distribution and decentralization; (iii) connectedness between different elements of HIV health services; (iv) outcomes and impact of service availability and connectedness at a population level; and (v) interaction between HIV service delivery and other parts of the health system. To meet our study objectives and the availability of existing data, we focused primarily on (ii) and (iii) above.

The service availability assessment focused on examining geographical distribution of services and service delivery decentralization. In assessing service connectedness, we defined four functions: 1) local coordination mechanisms and HIV outpatient clinic 'plus' (Hub and Heart of Continuum); 2) chronic care provision including self-care, peer support and patient follow-up and tracking as well as recording systems throughout the stages of HIV diagnosis, pre-ART care, ART and end-of-life care (Chronological Continuum); 3) linkages and/or integration across HIV and other health services, across different geographical administrative areas, and across community health facilities and closed settings such as detention centers for drug users and SWs and prisons (Horizontal Continuum); and 4) service linkages across commune, district and provincial levels including prevention outreach and $\mathrm{CHBC}$ (Vertical-Community Continuum).

\section{Literature search and review}

We conducted extensive review of HIV program and project reports, policy documents, legislation, and published articles. A primary source was the office of the Vietnam Authority (formerly 'Vietnam Administration') of HIV/ AIDS Control (VAAC), Ministry of Health. We also searched electronic databases such as Popline, Web of Science, Medline, Embase, and Google scholar. Using these sources, we identified articles published in English between January 1990 and August 2011. Keywords used for the search include HIV, AIDS, prevention, treatment, care, support and Vietnam in appropriate combinations and syntax according to the database. To fully explore the situation in Vietnam to the end of 2010, we also explored the reference lists of the key identified papers and consulted program staff of various organizations. We then analyzed the data obtained using the COPC analytical framework and identified strengths and constraints of HIV health service delivery in improving HIV case detection and retention in care.

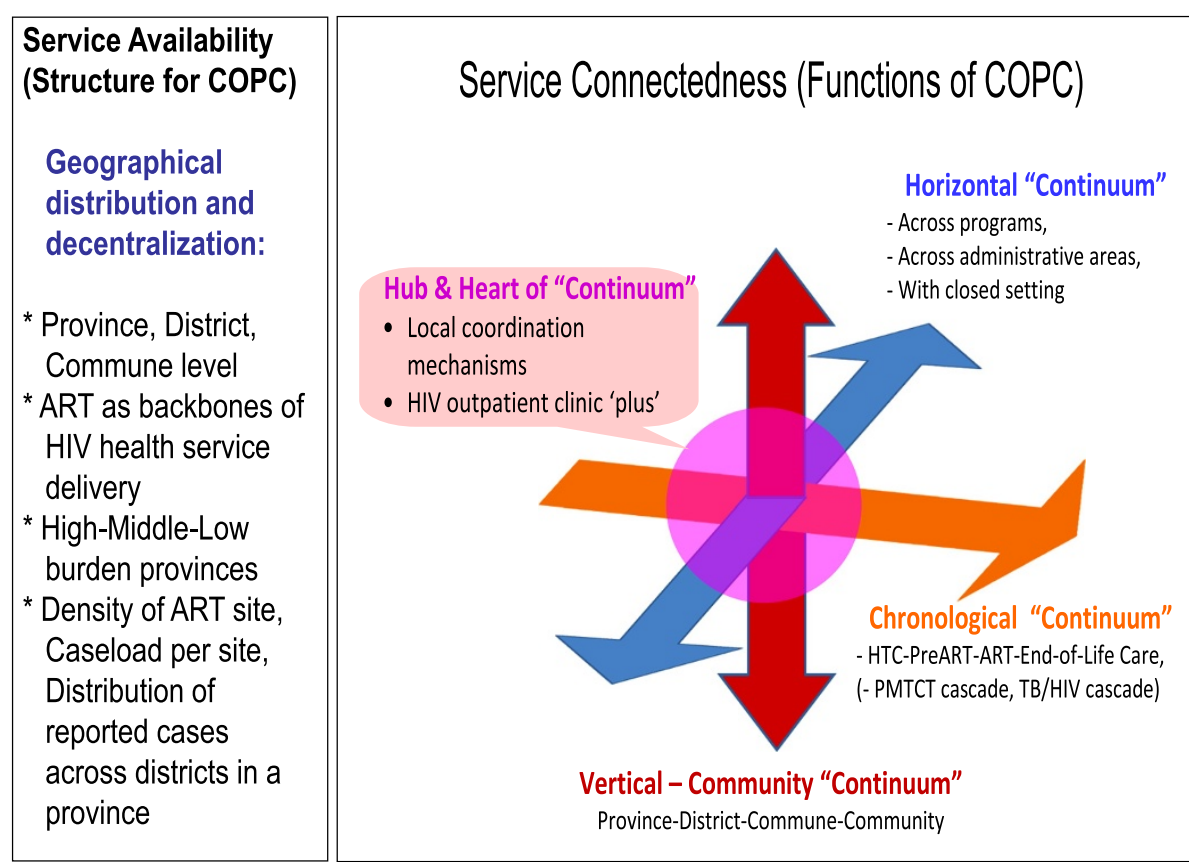

Figure 1 Framework for assessing HIV health services from the viewpoint of COPC. 


\section{Results}

Service availability including geographical distribution and decentralization

Organization of local health facilities follows the administrative divisions of 63 provinces, 697 districts and 11,112 communes [28]. TB diagnosis (smear) and delivery services were noted to be available in all districts. All communes were charged with providing TB treatment and antenatal care services.

HIV prevention for MARPs and HIV care and treatment has been rapidly expanded since 2004. Evolution of HIV health services are summarized in Table 1 [29-32]. By 2010 HIV prevention targeting MARPs included needle and syringe programs for IDUs. These programs were implemented in 3,333 communes in 352 districts or 51\% of all districts in 43 provinces mainly by 1,792 peer educators (Table 2) (VAAC: Unpublished report; 2010). Condom use program targeting SWs involved 3,123 peer educators. In addition, voluntary counseling and testing (VCT) and ART services co-existed in the same districts in most cases. That is, 175 districts had VCT and 167 of these districts had ART sites. At the provincial level, both VCT and ART were present in all provinces. PMTCT services involving antiretroviral prophylaxis were available in 133 districts in 63 provinces. Methadone maintenance therapy (MMT) was available in 11 districts in 4 provinces.

The density of ART sites in the provinces (number of ART sites / number of districts $\times 100$ ) was $66 \%$ in the high, $29 \%$ in the middle, and $15 \%$ in the low burden provinces (Table 3). It was noted that a number of high and middle burden provinces had mountainous remote areas where a substantive portion of MARPs and PLHIV had limited physical access to health facilities.

\section{Service connectedness}

1) Hub and heart of continuum

a) Local coordination mechanisms

Mechanisms for local coordination of HIV and other related health services were stipulated in several official documents from the Ministry of Health and its donorfunded projects. These documents included national guidance on HIV care and treatment, TB/HIV, PMTCT as well as project guidance on HIV prevention for MARPs [33-39]. Consequently, a variety of coordination committees for HIV-related services were formed in some areas, while none were formed in other areas. Most provinces lacked coordination mechanisms for HIV-related services between districts. That is, districts providing ART/VCT services rarely coordinated with districts without such services (Bui DD: Unpublished presentation; 2010).

b) HIV outpatient clinic 'plus'

The national guidance on HIV care and treatment stipulated the responsibilities undertaken in the HIV outpatient clinics [33,34]. These stipulations included the provision of clinical services, health education, prevention services, and psychosocial support. Other stipulations included the

Table 1 Evolution of HIV health services in Vietnam

\begin{tabular}{|c|c|}
\hline Year & Evolution \\
\hline Early 1990s & $\begin{array}{l}\text { Small-scale responses initiated at the local level such as local government led needle and syringe } \\
\text { programs and peer support activities. }\end{array}$ \\
\hline Mid 1990s & $\begin{array}{l}\text { The Ministry of Health initiated a HIV prevention campaign followed by commune health station based } \\
\text { basic care and support for PLHIV in three provinces. }\end{array}$ \\
\hline \multirow[t]{2}{*}{ Early 2000s } & $\begin{array}{l}\text { The } 100 \% \text { condom use program piloted by the Ministry of Health and a number of needle and syringe } \\
\text { programs implemented by non-governmental organizations. }\end{array}$ \\
\hline & $\begin{array}{l}\text { Establishment of HIV clinical services at national hospitals followed by district level HIV outpatient clinics } \\
\text { offering comprehensive care in Ho Chi Minh City. }\end{array}$ \\
\hline \multirow[t]{4}{*}{2004 (and onward) } & $\begin{array}{l}\text { Health sector-led large scale expansion of HIV prevention, care and treatment initiated } \\
\text { (supported by the United States, the United Kingdom, the World Bank and the Global Fund) }\end{array}$ \\
\hline & $\begin{array}{l}\text { For HIV care and treatment, HIV outpatient clinics expanded based on the experiences of a number of } \\
\text { model sites. }\end{array}$ \\
\hline & $\begin{array}{l}\text { Community- and home-based care (CHBC) expanded in different forms, such as (i) HIV outpatient clinic } \\
\text { based; (ii) Stand-alone model run by PLHIV groups, faith-based organizations or local non-governmental } \\
\text { organizations; (iii) Led by Women's Union; and (iv) Commune health station based. }\end{array}$ \\
\hline & ART expanded in administrative detention centers for IDUs and SWs, followed by in prisons. \\
\hline 2007 & $\begin{array}{l}\text { National Plan of Action on Harm Reduction approved. It stipulated that HIV officers at provincial and } \\
\text { district health services play a central role in mobilizing peer educators from current or former IDUs/SWs } \\
\text { and entertainment establishment owners/managers. }\end{array}$ \\
\hline 2008 & $\begin{array}{l}\text { Under the legal framework of the Law on HIV and its decree, the national pilot Methadone Maintenance } \\
\text { Therapy (MMT) program began in two provinces, }\end{array}$ \\
\hline
\end{tabular}

(Source: [29-32], Le TG: Unpublished presentation; 2005, Fujita M and Green K: Unpublished presentation; 2010, VAAC: Unpublished report, 2010). 
Table 2 Strengths and constraints of HIV health service delivery in improving HIV case detection and retention in care

\begin{tabular}{|c|c|c|}
\hline & Strengths & Constraints \\
\hline \multirow[t]{2}{*}{ Availability } & - Outreach peer educators in more than half of districts $[D, R]$ & $\begin{array}{l}\text { - Less than one-third of districts offering VCT/ART at district } \\
\text { level in middle/low HIV burden provinces }[D, R]\end{array}$ \\
\hline & $\begin{array}{l}\text { - Two-thirds of districts offering VCT/ART at district level in } \\
\text { high HIV burden provinces }[D, R]\end{array}$ & $\begin{array}{l}\text { - Lack of physically accessible VCT/ART in remote areas in } \\
\text { high/middle burden provinces }[D, R]\end{array}$ \\
\hline \multicolumn{3}{|l|}{ Connectedness } \\
\hline \multirow[t]{3}{*}{ Hub \& Heart } & $\begin{array}{l}\text { - Coordination mechanism between administrative detention } \\
\text { centers and HIV outpatient clinics emerging [R] }\end{array}$ & $\begin{array}{l}\text { - No coordination mechanism between districts with } \\
\text { VCT/ART and those without }[D, R]\end{array}$ \\
\hline & $\begin{array}{l}\text { - HIV outpatient clinic 'plus' at district level expanded in high } \\
\text { and middle burden provinces }[D, R]\end{array}$ & $\begin{array}{l}\text { - Clinical services only in government funded HIV outpatient } \\
\text { clinic at provincial level }[D, R]\end{array}$ \\
\hline & & - No system to monitor expansion of outpatient clinic 'plus' $[D, R]$ \\
\hline \multirow[t]{3}{*}{ Chronological } & $\begin{array}{l}\text { - Chronic care based ART case management established for } \\
\text { IDU and non-IDU [R] }\end{array}$ & $\begin{array}{l}\text { - Limited capacity to address the needs of PLHIV on ART } \\
\text { for many years [R] }\end{array}$ \\
\hline & - Palliative care initiated integrated with cancer care $[R]$ & - Pre-ART care under-developed [R] \\
\hline & & - Linkage from VCT to pre-ART care under-developed [R] \\
\hline \multirow[t]{2}{*}{ Horizontal } & $\begin{array}{l}\text { - HIV testing and counseling integrated into TB and antenatal } \\
\text { care in donor funded districts with ARTNCT in high } \\
\text { (and middle) burden provinces [D] }\end{array}$ & $\begin{array}{l}\text { - Lack of linkage for HIV-TB and HIV-MCH in non-donor funded } \\
\text { districts without VCT/ART in middle/low burden provinces [D] }\end{array}$ \\
\hline & $\begin{array}{l}\text { - Referral system between administrative detention centers } \\
\text { and HIV outpatient clinics being developed [R] }\end{array}$ & $\begin{array}{l}\text { - HIV service register not designed to facilitate TB/HIV and } \\
\text { PMTCT [R] }\end{array}$ \\
\hline \multirow[t]{3}{*}{ Vertical } & $\begin{array}{l}\text { - Extensive mobilization of peer educators to facilitate MARPs } \\
\text { to access VCT [D] }\end{array}$ & $\begin{array}{l}\text { - Access to HIV testing and care and treatment in advanced } \\
\text { stage of HIV infection }[D, R]\end{array}$ \\
\hline & - Alternative approaches to reach hidden MARPs emerging [D] & $\begin{array}{l}\text { - Health workers commonly providing verbal advice only to } \\
\text { patients for referral across different levels of health facilities }[R]\end{array}$ \\
\hline & - CHBC models mobilizing a wide range of stakeholders $[R]$ & - No system to monitor referral services $[R]$ \\
\hline
\end{tabular}

Remark:

[D] stands for a strength or constraint that is related to HIV case detection.

[R] stands for a strength or constraint that is related to retention in care.

provision of linkages with other relevant health services and the involvement of PLHIV as members of care teams and local HIV treatment committees.

Large donor funded initiatives supported expansion of HIV outpatient clinics mainly in high and middle burden provinces (Table 2). In addition to the clinical services, several functions were added consistent to support the concept of the HIV outpatient clinic 'plus'. These functions included: (i) facilitating HIV positive MARPs to access HIV outpatient clinics; (ii) involving HIV positive MARPs in HIV prevention initiatives targeting MARPs; (iii) supporting treatment adherence in collaboration with commune health stations, peer educators and CHBC teams; (iv) linking with $\mathrm{TB}$ and $\mathrm{MCH}$ services as well as closed settings; (v) establishing patient referral procedures to specialized hospitals [38-40]. Despite these initiatives, however, some HIV outpatient clinics were known to be only providing clinical services, especially in low burden provinces where limited donor funded projects were operating.

\section{2) Chronological continuum}

National guidance documents stipulated case management procedures and provided standardized longitudinal registers for pre-ART care and ART [41]. These documents were in line with the chronic care principles including selfcare, peer support, and patient follow-up information systems [42].

HIV outpatient clinics tended to actively prepare and track patients for ART by mobilizing PLHIV peer support and CHBC. Program data indicated the percentage of adults and children with HIV still alive and known to be on treatment 12 months after initiation of ART was $84.2 \%$ [43]. HIV drug resistance early warning indicators indicated good adherence to appointment schedule and low level of lost-to-follow-up despite a large proportion of the patients being IDUs (Figure 2-a) [44,45]. Furthermore, a study conducted a 2-year prospective cohort analysis of patients taking ART in two HIV outpatient clinic 'plus' sites in Ho Chi Minh City. It revealed the change of median CD4 count over the 24-month follow-up period among patients who ever injected illicit opiates was similar to that for those who reported never having injected [46]. In another study in Hanoi [47] viral suppression was not statistically different among the patients who used drug in the previous six months versus those who did not use it after at least six months of ART initiation.

To meet palliative care needs [48], the Ministry of Health [49] developed the guidelines on palliative care for cancer and AIDS patients in 2006. The ministry also improved 
Table 3 Availability of ART sites according to different levels of HIV burden in 2009

\begin{tabular}{|c|c|c|c|c|c|c|}
\hline & \multicolumn{2}{|c|}{ High burden province } & \multicolumn{2}{|c|}{ Middle burden province } & \multicolumn{2}{|c|}{ Low burden province } \\
\hline & $\mathbf{n}$ & $(\%)$ & $\mathbf{n}$ & $(\%)$ & $\mathbf{n}$ & $(\%)$ \\
\hline Number of provinces & 8 & & 29 & & 26 & \\
\hline Number of districts & 122 & & 300 & & 268 & \\
\hline Number of ART sites & 80 & & 88 & & 39 & \\
\hline District with ART & 65 & & 72 & & 30 & \\
\hline Density of ART site (\%) & & 65.6 & & 29.3 & & 14.6 \\
\hline \multicolumn{7}{|l|}{ (Number of ART sites / Number of districts x 100) } \\
\hline Estimated number of patients needing ART & 36,682 & $(58.0)$ & 21,197 & (33.5) & 5,409 & $(8.5)$ \\
\hline Number of patients on ART & 25,449 & $(70.7)$ & 8,464 & $(23.5)$ & 2,095 & $(5.8)$ \\
\hline Estimated number of patients needing ART per district & 301 & & 71 & & 20 & \\
\hline Number of ART patients per district & 209 & & 28 & & 8 & \\
\hline \multicolumn{7}{|l|}{ ART coverage (\%) } \\
\hline $\begin{array}{l}\text { (Number of patients on ART / Estimated number } \\
\text { of patients needing ART } \times 100 \text { ) }\end{array}$ & & 69.4 & & 39.9 & & 38.7 \\
\hline
\end{tabular}

opioid prescribing regulations in 2008 and trained over 400 physicians by early 2010 [50]. As a result, palliative care services have started in both hospitals and communities [51].

Patient follow-up and tracking for pre-ART care appeared to be considerably less operational than for ART. Results from an ad hoc assessment indicated that a substantial number of PLHIV might be dying or lostto-follow-up during the pre-ART period (Figure 2-b) (Hanoi Health Services HIV/AIDS Center: Unpublished record review; 2010). In 2009, monitoring of attrition from pre-ART care started as part of HIV drug resistance early warning indicators monitoring [52].

Existing referral forms were not used extensively to facilitate the referral process from VCT to pre-ART care. An ad hoc assessment in one province indicated a large gap between the number of people diagnosed as HIV positive at VCT and the number of people enrolled in pre-ART care (Figure 2-c) (Kato M: Unpublished presentation; 2010). No specific national guidance or patient tracking system was established for this process.

\section{3) Horizontal continuum}

Collaborative activities between HIV and TB services and $\mathrm{HIV}$ and MCH services (PMTCT) were expanded mainly through donor funded projects. These collaborative efforts were consistent with the national guidance developed by concerned national programs [35,36] (Table 2).

However, these projects tended to support specific districts rather than a provincial network. In districts without donor-funded VCT/ART, scarcely any health workers were charged with providing HIV health services
(COPC review group: Unpublished report, 2010). In these districts, TB and antenatal care services were rarely equipped to implement provider-initiated testing and counseling for TB cases and for pregnant women. As a result, in 2009 the percentage of estimated HIV-positive incident TB cases that received treatment for TB and HIV was as low as $27.5 \%$ [43]. Similarly, the percentage of HIV-positive pregnant women who received antiretroviral medicines to reduce the risk of mother-to-child transmission was $32.3 \%$.

Standard TB registers included a section for recording HIV status. However, antenatal care registers did not include a space to record HIV status among pregnant women. Pre-ART and ART registers did not have a section for recording TB diagnosis and treatment or for pregnancy status. Patient-held records provided this additional information but were used in only a limited number of sites.

All the methadone maintenance therapy (MMT) sites were located in districts where VCT/ART was present [53] (VAAC: Unpublished report; 2010). Some of the current MMT sites were stand-alone and physically separate from HIV outpatient clinics while others were co-located with HIV outpatient clinics. Efforts were made to strengthen linkages between MMT and other HIV health services as part of the MMT scale-up.

4) Vertical continuum

a) Linkages across different levels of health services

PLHIV suspected of active TB were often required to travel long distances across the community, district, and provincial levels. Diagnosis of smear-negative and extrapulmonary TB was mostly performed at the provincial 


\begin{tabular}{|l|c|c|c|}
\hline \multicolumn{1}{|c|}{ Indicators } & Target & $\begin{array}{c}\text { Adult sites } \\
\text { meeting } \\
\text { suggested } \\
\text { targets }\end{array}$ & $\begin{array}{c}\text { Pediatric sites } \\
\text { meeting } \\
\text { suggested } \\
\text { targets }\end{array}$ \\
\hline $\begin{array}{l}\text { \% appropriate initial ART regimen } \\
\text { prescriptions (Jan-Dec 2008) }\end{array}$ & $\mathbf{1 0 0 \%}$ & $\begin{array}{c}\mathbf{2 5 / 2 7} \\
\mathbf{( 9 2 . 6 \% )}\end{array}$ & $\begin{array}{c}\mathbf{3 / 4} \\
\mathbf{( 7 5 \% )}\end{array}$ \\
\hline $\begin{array}{l}\text { \% lost to follow up during the first 12 } \\
\text { months of ART (Jan-Dec 2007) }\end{array}$ & $\leq \mathbf{2 0 \%}$ & $\begin{array}{c}\mathbf{2 6 / 2 7} \\
\mathbf{( 9 6 . 3 \% )}\end{array}$ & $\begin{array}{c}\mathbf{4 / 4} \\
\mathbf{( 1 0 0 \% )}\end{array}$ \\
\hline $\begin{array}{l}\text { \% ART patients keeping all clinical } \\
\text { appointments on time (Jul-Sep 2008) }\end{array}$ & $\geq \mathbf{8 0 \%}$ & $\begin{array}{c}\mathbf{2 0 / 2 5} \\
\mathbf{( 8 0 \% )}\end{array}$ & $\begin{array}{c}\mathbf{3 / 4} \\
\mathbf{( 7 5 \% )}\end{array}$ \\
\hline $\begin{array}{l}\text { \% retained on first-line ART at 12 } \\
\text { months (Jan-Dec 2007) }\end{array}$ & $\geq \mathbf{7 0 \%}$ & $\mathbf{2 2 / 2 7}$ & $\mathbf{4 / 4}$ \\
$\mathbf{( 8 1 . 5 \% )}$ & $\mathbf{( 1 0 0 \% )}$ \\
\hline
\end{tabular}

2-a. Results of HIV drug resistance early warning indicators (Source: $[44,45]$ )

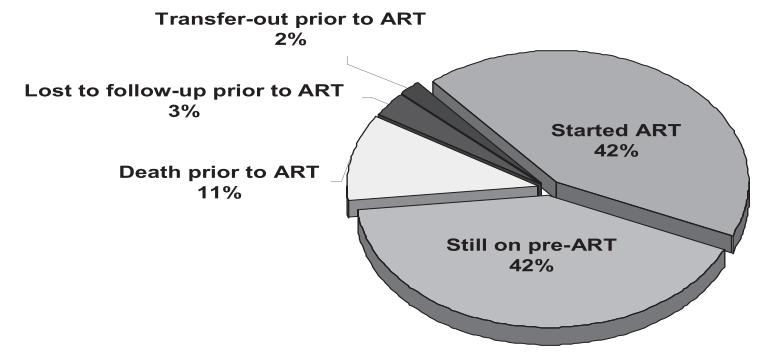

2-b. Status of PLHIV enrolled in pre-ART care from January 2008 to June 2009 at 3 outpatient clinics in Hanoi as of June 2009 (Source: Hanoi Health Services HIV/AIDS Center: Unpublished record review; 2010)

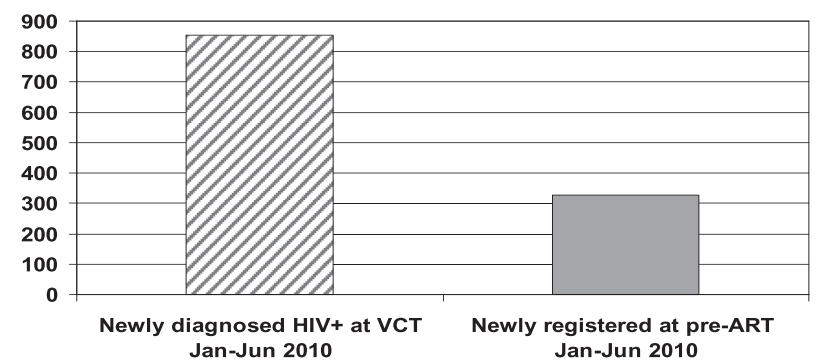

2-c. Number of people diagnosed HIV-positive reaching outpatient clinics in Thanh Hoa Province (Source: Kato M: Unpublished presentation; 2010)

Figure 2 Outcomes of people diagnosed HIV-positive and initiated ART.

level. Similarly, a number of PMTCT services were provided mainly at the provincial level. These services included planned delivery of HIV positive women, early infant diagnosis and pediatric treatment. In addition, the provincial level was responsible for confirmation of HIV treatment failure and prescription of second line ART regimens in most provinces [41].
All of these patient flows required robust referral systems including patient information sharing across the different levels. Commonly, a doctor simply instructed a patient to go to another facility often without a referral form (Table 2). There were no routine mechanisms to monitor the functioning of the referral process (COPC review group: Unpublished report; 2010). 
Respect for administrative boundaries and the hierarchy of authority were reported to often make health workers reluctant to contact their peers in other health facilities.

b) Community response and its linkage with health services

Peer educators for condom use and needle and syringe program were encouraged to systematically refer their clients to VCT (Table 2). An increasing number of MSM peer educators were also recruited particularly in large cities. However, the coverage of HIV prevention programs for male IDUs, female SWs and MSM was only $15.4 \%, 47.3 \%$ and $24 \%$, respectively in 2009 [43]. Similarly, the coverage of HIV testing and counseling among IDUs, female SWs and MSM was low at $17.9 \%$, $34.8 \%$ and $19.1 \%$, respectively. Consequently, majority of PLHIV accessed HIV care and treatment services at an advanced stage of HIV infection. Program monitoring data from 2009 indicated 64\% of PLHIV started ART at CD4 $100 / \mathrm{cm}^{3}$ or lower [52].

Alternatives to the peer education approach were reported to be emerging to serve hard-to-reach populations. These alternatives included mobilization of pharmacies, street vendors, self-service boxes and commune health stations for needle and syringe and condom use programs [54]. Civil society partners also began to extend their reach to MSM through internet connections and cruising hot spots.

In most districts, peer educators for prevention and those for care were reported to be managed and supported separately by different donor funded projects. However, there were growing examples of synergy between the activities of the two different peer educator groups [54]. These examples included drop-in-centers for IDUs managed by PLHIV with support from district HIV outpatient clinics, and needle and syringe program activities performed by PLHIV.

\section{Discussion}

\section{Service availability including geographical distribution} and decentralization

Only a quarter of districts had VCT or ART sites, while MARPs had been identified and reached in more than half of the districts. TB diagnosis (smear) and antenatal care services were provided in all the districts throughout the country.

In high burden provinces, estimated ART coverage appeared to be reaching saturation. However, in middle and low burden provinces, the coverage appeared to be relatively low and the ART caseload per facility was too small to warrant further expansion of district ART sites. In some remote areas rated high and middle burden, a substantive number of PLHIV have limited physical access to district health facilities.

To maximize HIV case detection and retention in care, it is therefore crucial to strengthen service connectedness in middle and low burden provinces. Remote areas in high and middle burden provinces require consideration of specific approaches. Such initiatives include further decentralized and/or mobile services $[55,56]$.

\section{Service connectedness}

\section{1) Local coordination mechanisms}

Local coordination mechanisms for HIV-related health services vary significantly across different provinces and in some cases they are non-existent. It would be beneficial to review the experiences from different forms of coordination mechanisms across the country to inform future guidance development [40]. In particular, consideration should be given to coordination mechanisms between districts with VCT/ART and those without. One option is to form clusters of districts in each province and to establish clear referral procedures as implemented in a country in Asia [57]. In this way, within the same cluster, districts offering VCT/ART could collaborate with districts not offering VCT/ART services to improve HIV case detection and retention in care.

\section{2) Functions of HIV outpatient clinic}

HIV outpatient clinics established multiple functions to the chronological, horizontal and vertical continuum of prevention and care. This was accomplished through mobilizing PLHIV and MARPs peer educators and by developing links to $\mathrm{CHBC}$ and other related services as seen in other Asian countries [18,40]. This was particularly evident in high burden provinces.

Early in the epidemic, PLHIV and MARPs were seen as passive recipients of services. Over the past decade, there has been a paradigm shift. When new HIV outpatient clinics were established, health workers encouraged PLHIV and MARPs to work as peer educators, care providers and support-group members. This provided opportunities for health workers to receive feedback on their services. As a result, acceptance and trust of health services among PLHIV and MARPs have improved. This improved relationship facilitated a rapid expansion of peer educators.

However, limited information was available to know the status of more than 160 HIV outpatient clinics across the country. Establishment of a simple system to monitor the functions of these HIV outpatient clinics would help national and local HIV programs to improve 
HIV case detection and retention in care especially among MARPs.

\section{3) Chronic care}

Chronic care systems for PLHIV on ART were well established through peer mobilization, patient followup and tracking, and longitudinal monitoring $[42,58]$. Their effectiveness is reflected in high levels of ART retention and appointment keeping within a twentyfour month period. Such success is evident despite the fact that a large proportion of the patients are IDUs without MMT services [46,47].

However, retention from HIV testing and counseling to pre-ART care and during pre-ART care were suboptimal as in the case in Sub-Saharan Africa [59]. These processes should be strengthened to accelerate early ART initiation and to introduce ART as prevention among discordant couples $[2,60]$. It will also improve case management of TB-HIV co-infections and HIV positive pregnant women. Integrating pre-ART care with post-test counseling services should be considered $[61,62]$ as VCT and ART sites were co-located in most cases in Vietnam.

It is also vital to establish the mechanisms to assess and address the needs of PLHIV on ART over the long term. These PLHIV include those who are mobile across provinces and those who have mental health problems, cardiovascular diseases, cancers and viral hepatitis $B$ and $C[5,6]$.

4) Linkages across different services, administrative boundaries and settings

Integration of HIV testing and counseling into TB and antenatal care services is progressing in districts offering VCT/ART through donor-funded projects. In districts without VCT/ART, no mechanisms appeared to be in place to facilitate access to HIV testing and counseling and to support retention in care.

One option for consideration is to form district clusters [57]. In addition, districts without VCT/ART should explore new approaches to introduce HIV testing and counseling for MARPs, TB cases and pregnant women. Such approaches might include point-of-care HIV diagnosis with rapid-test based algorithms and community outreach testing and counseling [55,56].

The introduction of ART in administrative detention centers has led to the referral system development between these centers and hospitals in the community. It has also triggered the potential of strengthening the basic health care in closed settings including prisons into the coming time.

5) Linkages across different levels of health services
The existing patient flows for TB/HIV, PMTCT and HIV treatment failure management require robust referral systems across the different levels of health services. However, limited information was available to assess the functioning of the referral processes. A simple monitoring system should be developed to assess retention including drop-out and delay during the referral processes.

Administrative boundaries and the hierarchy of authority appear to hinder effective referral services. People-centered health care has been promoted in Asian countries including Vietnam [63]. This approach could help overcome some of the challenges to patient referral.

6) Community response and its linkage with health services

Extensive peer educator schemes established by local health services can be regarded as a major breakthrough in reaching and serving hard-to-reach populations and to ensure adherence to HIV treatment. These schemes are particularly critical in Vietnam where the existence of non-government organizations is limited.

However, a substantial portion of MARPs seems to be still hidden resulting in limited and delayed access to HIV services. Considerations should be given to expanding alternative approaches such as the mobilization of pharmacies and street vendors [54]. Also the capacity of existing peer educators should be maximized. For example, approaches to be considered include promoting synergy between the two schemes of peer educators for prevention and care [64]. Strategies to further decentralize HIV testing and counseling and care and treatment services should be explored. These strategies should include mobile services in the areas where a substantive number of PLHIV have limited physical access to services. These efforts would improve HIV case detection and retention in care and contribute to better outcomes of multiple HIV health services including targeted prevention, TB/HIV, PMTCT and MMT.

\section{Utility of the COPC framework}

This systematic assessment of HIV health service delivery using the COPC framework identified underlying system-related strengths and constraints which affect the performance of multiple HIV health services, particularly HIV case detection and retention in care. Lessons learned from this review could contribute to the optimization of service delivery and the adaptation of Treatment 2.0 to Vietnam's specific situation $[8,9]$.

While this review focused on HIV health service delivery, the COPC framework could also serve to systematically assess the interfaces between HIV and other health service delivery systems. This is particularly important in 
the context of the global health agenda to shift from disease control programs to health system strengthening. Such interface could include mechanisms for coordinating and linking different health services, cross-fertilization of management of various chronic illnesses [58], management of patient referral systems, and community mobilization and outreach.

\section{Limitations}

This review is a first attempt to capture the wide range of HIV service delivery processes by using the COPC framework. However, not every element of HIV health services has been addressed. For instance, services for children born to HIV positive mothers have not been included in this analysis. Another limitation of this study is that it includes the analysis of unpublished documents. However, some of the coauthors were involved in the national program from its inception and played important roles in policy development and service delivery. With this knowledge and experience, the authors were able to critically discuss and synthesize each issue presented in the paper.

\section{Conclusions}

This study identified the system-related strengths and constraints of HIV health service delivery in maximizing HIV case detection and retention in care in Vietnam. District-based service delivery models have been developed especially in high burden provinces. Multiple service elements appeared to be connected well in districts offering VCT/ART where donor funded projects were operating. Extensive involvement of PLHIV and MARPs in prevention and care lowered barriers for the marginalized populations to access services. However, service elements tended to be disconnected in districts that were not supported by donor funded projects particularly in middle and low burden provinces. In addition, no adequate service delivery model has been established for MARPs and PLHIV in mountainous remote areas. Based on this review, the national HIV program has initiated pilot projects to address the constraints identified. Furthermore, it plans to strengthen provincial programming by re-defining various service linkages and accelerate the transition from project-based approach to integrated service delivery in line with the Treatment 2.0 initiative. Similar reviews should be considered by the national HIV programs of other countries to optimize respective HIV service delivery models.

\section{Abbreviations}

ART: Antiretroviral treatment; CHBC: Community- and home-based care; COPC: Continuum of prevention and care; HTC: HIV testing and counseling; IDUs: Injecting drug users; MSM: Men who have sex with men; MARPs: Mostat-risk populations; MCH: Maternal and child health; MMT: Methadone maintenance therapy; PLHIV: People living with HIV; PMTCT: Prevention of mother-to-child transmission; SWs: Sex workers; TB: Tuberculosis;
VAAC: Vietnam Authority of HIV/AIDS Control; VCT: Voluntary counseling and testing.

\section{Competing interests}

The authors declare that they have no competing interests except that KCP has received remuneration from World Health Organization Vietnam to prepare a preliminary draft on the overview of HIV health services in Vietnam.

\section{Authors' contributions}

MF and KCP designed the study, coordinated the collection, analysis and interpretation of data and drafted the manuscript. MK, NTMT and DJ participated in design of the study, analysis and interpretation of data and drafting of the manuscript. KG contributed to data collection and revisions of the entire manuscript. DTN, BDD, NVK, CTTT, NTL contributed to conceptualization of the study, participated in data collection and provided inputs to the manuscript. MJ contributed to refinement of methods, interpretation of data and revisions of the manuscript. All authors read and approved the final manuscript.

\section{Authors' information}

MF and KG were with World Health Organization Vietnam and FHI 360 Vietnam respectively during the study period.

\section{Acknowledgements}

The authors would like to thank Dr Ying-Ru Lo, Dr Anne Johnson and Dr Elizabeth Lindsey for providing comments on draft versions of this article. They also express appreciation to Dr Nguyen Thi Thuy Van, Ms Nguyen Thien Nga, Dr Fabio Mesquita, Dr Teodora Wi and to staff of the Care and Treatment, Harm Reduction and Surveillance, Monitoring and Evaluation Departments of VAAC, Global Fund HIV Project, the United States President's Emergency Plan for AIDS Relief Vietnam (the United States Agency for International Development and the United States Centers for Disease Control and Prevention), HIV/AIDS Prevention and Control in Vietnam Project funded by the World Bank and the United Kingdom Department for International Development, provincial AIDS centers, health facilities at district and commune levels, and FHI 360 Vietnam and other partners of the United States President's Emergency Plan for AIDS Relief for providing information and data and supporting this review. The extensive contributions of PLHIV and MARP peer educators are greatly appreciated.

The views expressed in this paper are those of the authors and do not necessarily represent the official views of their organizations.

\section{Author details}

'World Health Organization Cambodia Office, P.O. Box 1217, No. 177-179 Pasteur (St.51) (corner 254) Sangkat Chak Tomouk, Phnom Penh, Cambodia. ${ }^{2}$ Department of Public Health, School of Public Health and Health Sciences, University of Massachusetts Amherst, 316 Arnold House, 715 North Pleasant St, Amherst, MA01003-9304, USA. Vietnam Authority of HIV/AIDS Control, Ministry of Health Vietnam, 135/3 Nui Truc, Ba Dinh, Hanoi, Vietnam. ${ }^{4}$ National Hospital of Tropical Diseases, 78 Giai Phong Street, Hanoi, Vietnam. ${ }^{5} \mathrm{FHI}$ 360, Demmco House, 1st Dzorwulu Crescent, Accra, Ghana. ${ }^{6}$ World Health Organization Vietnam Office, 63 Tran Hung Dao, Hoan Kiem District, P.O. Box 52, Hanoi, Vietnam. ${ }^{7}$ Department of Community and Global Health, Graduate School of Medicine, The University of Tokyo, 7-3-1 Hongo,

Bunkyo-ku, Tokyo, Japan.

Received: 6 February 2012 Accepted: 24 November 2012 Published: 29 December 2012

\section{References}

1. Lawn SD, Harries AD, Anglaret X, Myer L, Wood R: Early mortality among adults accessing antiretroviral treatment programmes in sub-Saharan Africa. AIDS 2008, 22:1897-1908.

2. Cohen MS, Chen YQ, McCauley M, Gamble T, Hosseinipour MC, Kumarasamy N, Hakim J, Kumwenda J, Grinsztejn B, Pilotto JHS, Godbole SV, Mehendale S, Chariyalertsak S, Santos BR, Mayer KH, Hoffman IF, Eshleman SH, Piwowar-Manning E, Wang L, Makhema J, Mills L, de Bruyn G, Sanne I, Eron J, Gallant J, Havlir D, Swindells S, Ribaudo H, Elharrar V, Burns D, et al: Prevention of HIV-1 infection with early antiretroviral therapy. New Engl J Med 2011, 365:493-505. 
3. Joint United Nations Programme on HIV/AIDS: Getting to Zero: 2011-2015 Strategy. Geneva: Joint United Nations Programme on HIV/AIDS; 2010.

4. Care and Support Working Group of the UK Consortium on AIDS and International Development: Care \& Support: The forgotten pillar of the HIV response. London: UK Consortium on AIDS and International Development; 2011.

5. Gilks CF, Crowley S, Ekpini R, Epkini R, Gove S, Perriens J, Souteyrand Y, Sutherland D, Vitoria M, Guerma T, De Cock K: The WHO public-health approach to antiretroviral treatment against HIV in resource-limited settings. Lancet 2006, 368:505-510.

6. Joshi D, O'Grady J, Dieterich D, Gazzard B, Agarwal K: Increasing burden of liver disease in patients with HIV infection. Lancet 2011, 377(9772):1198-1209.

7. Commission on AIDS in Asia: Redefining AIDS in Asia: crafting an effective response. New Delhi: Oxford University Press; 2008.

8. Hirnschall G, Schwartlander B: Treatment 2.0: catalyzing the next phase of scale-up. Lancet 2011, 378:209-211.

9. World Health Organization and Joint United Nations Programme on HIV/ AIDS: The Treatment 2.0 Framework for Action: Catalysing the Next Phase of Treatment, Care and Support. Geneva: World Health Organization; 2011.

10. Srikantiah P, Ghidinelli M, Bachani D, Chasombat S, Daoni E, Mustikawati DE, Nhan DT, Pathak LR, San KO, Vun MC, Zhang F, Lo YR, Narain JP: Scale-up of national antiretroviral therapy programs: progress and challenges in the Asia Pacific region. AIDS 2010, 24(Suppl 3):S62-S71.

11. Legido-Quigley H, Montgomery CM, Khan P, Fakoya A, Getahun H, Grant A: Integrating tuberculosis and HIV services in low- and middle- income countries: A systematic review. Montreux, Switzerland: Background paper for the Global Symposium on Health Systems Research; 2010.

12. World Health Organization, United Nations Children's Fund, United Nations Population Fund and Joint United Nations Programme on HIV/AIDS: AsiaPacific operational framework for linking HIVISTI services with reproductive, adolescent, maternal, and newborn and child health services. Geneva: World Health Organization; 2008.

13. World Health Organization, United States Agency for International Development and Family Health International: Strategic considerations for strengthening the linkages between family planning and HIV/AIDS policies, programs, and services. Geneva: World Health Organization; 2009.

14. Narain JP, Chela C, van Praag E: Planning and Implementing HIV/AIDS Care programmes: A step-by-step approach. New Delhi: World Health Organization Regional Office for South-East Asia; 1998.

15. National Center for HIV/AIDS, Dermatology and STD (NCHADS), Ministry of Health Cambodia: The continuum of care for people living with HIV/AIDS: operational framework in Cambodia. Phnom Penh: National Center for HIV/ AIDS, Dermatology and STD, Ministry of Health; 2003.

16. World Health Organization Regional Office for the Western Pacific: HIV/AIDS care and treatment: guide for implementation. Manila: World Health Organization Regional Office for the Western Pacific; 2004.

17. World Health Organization Regional Office for the Western Pacific and National Center for HIV/AIDS, Dermatology and STD (NCHADS): The continuum of care for people living with HIV/AIDS in Cambodia: linkages and strengthening in the public health system. Case study. Manila: World Health Organization Regional Office for the Western Pacific; 2006.

18. Green K, McPherson R, Fujita M, Lo YR, Natpratan C, van Praag E, Gulaid L, Parker C: Introduction to the continuum of care. In Scaling up the continuum of care for people living with HIV in Asia and the Pacific: a toolkit for implementers. Bangkok: Family Health International; 2007:7-18.

19. Vietnam Administration of HIV/AIDS Control: Chapter V Results. In Vietnam HIV/AIDS Estimates and Projections 2007-2012. Hanoi, Vietnam: Ministry of Health; 2009:66-69.

20. Hammett TM, Wu Z, Duc TT, Stephens D, Sullivan S, Liu W, Chen Y, Ngu D, Jarlais DCD: 'Social evils' and harm reduction: the evolving policy environment for human immunodeficiency virus prevention among injecting drug users in China and Vietnam. Addiction 2007, 103:137-145.

21. Liebowitz B, Brody EM: Integration of research and practice in creating a continuum of care for the elderly. Gerontology 1970, 10:11-17.

22. Anderson S: Community responses to AIDS. World Health Forum 1994, 15:35-38.

23. Jackson H, Kerkhoven R: Developing AIDS care in Zimbabwe: a case for residential community centers? AIDS Care 1995, 7:663-686.

24. Schietinger H, Sanei L: A continuum of HIV/AIDS prevention and care. In Systems for delivering HIV/AIDS care and support, Discussion Paper on HIVI
AIDS Care and Support No.8. Arlington: Health Technical Services (HTS) Project; 1998:17-20.

25. International HIV/AIDS Alliance: Linking prevention and care. In Care, Involvement and Action: Mobilising and supporting community responses to HIVIAIDS care and support in developing countries. London: International HIV/ AIDS Alliance; 2000:6-7.

26. United Nations Children's Fund: Report 2006 East Asia and Pacific Regional Consultation on Children and HIV/AIDS, Hanoi, Vietnam. Bangkok: United Nations Children's Fund East Asia and Pacific Regional Office; 2006.

27. Green K, McPherson R, Fujita M, Lo YR, Natpratan C, van Praag E, Gulaid L, Parker C: Make it happen at the local level: Establishing the continuum of care. In Scaling up the continuum of care for people living with HIV in Asia and the Pacific: a toolkit for implementers. Bangkok: Family Health International; 2007:27-69.

28. General Statistics Office of Vietnam: Administrative divisions, population and labour. In Statistical Handbook of Vietnam. Hanoi: Statistical Publishing House; 2010:5-40.

29. National Committee for AIDS, Drugs and Prostitution (NCADP): Organization of Implementation. In The national strategy on HIV/AIDS prevention and control in Vietnam till 2010 with a vision to 2020. Hanoi: Medical Publishing House; 2004:186-189.

30. Assembly N: Law on HIV/AIDS Prevention and Control. Hanoi: National Assembly; 2006.

31. Government of Vietnam: Government's Decree No. 108/2007/ND-CP of June 26, 2007, Detailing the Implementation of a Number of Articles of the Law on HIV/AIDS Prevention and Control. Hanoi: Government of Vietnam; 2007.

32. Vietnam Administration of HIV/AIDS Control, Family Health International Vietnam, the United States President's Emergency Fund for AIDS Relief Viet Nam: Community and home-based care in Vietnam: Findings and recommendations from a rapid assessment. Hanoi: Family Health International; 2009

33. Ministry of Health: Contents of the National Action Plan. In National action plans on HIV/AIDS care and treatment to the year 2010. Edited by. Hanoi: Ministry of Health; 2007:22-28.

34. Ministry of Health: Part IV: Implementation Organization. In Antiretrovirus treatment protocol for people living with HIV/AIDS. Hanoi: Ministry of Health; 2007:11-12.

35. Ministry of Health: Part III: Implementation Organization. In Collaborative Protocol for TB/HIV Diagnosis, Treatment and Case Management. Hanoi: Ministry of Health; 2007:3-6.

36. Ministry of Health: Chapter III: Organization of Implementation. In Procedure of Care and Treatment of Mother-to-Child Transmission of HIV. Hanoi: Medical Publishing House; 2007:51-58.

37. Ministry of Health: Part IV: Organization of Implementation. In National Action Plan on Harm Reduction Intervention in HIV Prevention in 2007-2010 Period. Hanoi: Ministry of Health; 2007:25-28.

38. Country Coordinating Mechanism in Vietnam: 4.6.3 Activities. In HIV Proposal for Round 6 Funding for the Global Fund to Fight Against AIDS, TB and Malaria. Hanoi, Vietnam: Country Coordinating Mechanism in Vietnam; 2006:30-35.

39. Country Coordinating Mechanism in Vietnam: 4.5 Implementation Strategy. In HIV Proposal for Round 9 Funding for the Global Fund to Fight Against AIDS, TB and Malaria. Hanoi, Vietnam: Country Coordinating Mechanism in Vietnam; 2009:16-28.

40. Green K, McPherson R, Fujita M, Lo YR, Natpratan C, van Praag E, Gulaid L, Parker C: Continuum of care country profiles. In Scaling up the continuum of care for people living with HIV in Asia and the Pacific: a toolkit for implementers. Bangkok: Family Health International; 2007:19-26.

41. Part II: Management of ART protocol for people living with HIV/AIDS, Ministry of Health: Part II: Management of ART protocol for people living with HIV/AIDS. In Antiretrovial treatment protocol for people living with HIVI AIDS. Hanoi: Ministry of Health; 2007:2-7.

42. Bodenheimer $\mathrm{T}$, Wagner $\mathrm{EH}$, Grumbach $\mathrm{K}$ : Improving primary care for patients with chronic illness: the chronic care model, Part 2. JAMA 2002, 288:1909-1914.

43. Socialist Republic of Vietnam: National Response. In The fourth country report on Following up the implementation to the declaration of commitment on HN and AIDS: Reporting Period January 2008 - December 2009. Hanoi; 2010:11-32.

44. Vietnam Authority of HIV/AIDS Control: Report on outcome of ART cohort and pilot of HIV drug resistance early warning indicators data collection. Hanoi: Vietnam Authority of HIV/AIDS Control; 2010. 
45. Do TN, Nguyen TMT, Do MH, Kato M, Cao TT, Nguyen TTV, Nguyen VK, Bui DD, Nguyen TL, Fujita M: Service Delivery Site Factors are Associated with Antiretroviral Therapy Cohort Outcomes - Analysis of National Assessment Data to Inform Public Health Action [abstract]. In Abstract book of the $18^{\text {th }}$ Conference on Retroviruses and Opportunistic Infections. Boston, USA; 2011:284. http://www.retroconference.org/2011/Abstracts/ 41313.htm.

46. Tran VH, Nguyen NNT, Arnolda GRB, Burdon R, Green K, Mills S: Section 1: Cohort Study Results. In Results of the program evaluation of patients initiating antiretroviral therapy in two health facilities in Ho Chi Min City, Vietnam. Edited by. Hanoi: Family Health International; 2010:25-64.

47. Jordan MR, La H, Nguyen HD, Sheehan H, Lien TT, Duong DV, Hellinger J, Wanke C, Tang AM: Correlates of HIV-1 viral suppression in a cohort of HIV-positive drug users receiving antiretroviral therapy in Hanoi, Vietnam. International Journal of STDs \& AIDS 2009, 20:418-422.

48. Green $K$, Kinh $L N$, Khue $L N$ : Palliative care in Vietnam: findings from a rapid situation analysis in five provinces. Hanoi: Ministry of Health; 2006.

49. Ministry of Health: Guidelines on palliative care for cancer and AIDS patients. Hanoi: Medical Publishing House; 2006.

50. Krakauer EL, Cham NT, Khue LN: Vietnam's palliative care initiative: successes and challenges in the first five years. J Pain Symptom Manage 2010, 40:27-30.

51. Green K, Tuan T, Hoang TV, Trang NNT, Ha NTT, Hung ND: Integrating palliative care into HIV outpatient clinical settings: preliminary findings from an intervention study in Vietnam. J Pain Symptom Manag 2010, 40:31-34.

52. Nguyen TMT, Do TN, Kato M, Bui DD, Nguyen HH, Doan LTT, Nguyen TTV, Mesquita F: Integrating pre-ART and ART indicators in routine national data collection to improve HIV care and treatment outcome in Vietnam [abstract]. In Abstract Book of the 10 International Congress on AIDS in Asia and the Pacific. Busan, Korea; 2011:95.

53. Ministry of Health: Evaluation of the pilot model for the substitution treatment of opiate dependence using methadone in Haiphong and Ho Chi Minh City. Hanoi: Ministry of Health; 2009.

54. Fujita M, Zhao P, Jacka D: Targeted HIV prevention for injecting drug users and sex workers. Vietnam's first large-scale national harm reduction initiative. Hanoi, Vietnam: World Health Organization Country Office for Vietnam; 2009.

55. Sweat M, Morin S, Celentano D, Mulawa M, Singh B, Mbwambo J, Kawichai S, Chingono A, Khumalo-Sakutukwa G, Gray G, Richter L, Kulich M, Sadowski A, Coates T, the Project Accept study team: Community-based intervention to increase HIV testing and case detection in people aged $16-32$ years in Tanzania, Zimbabwe, and Thailand (NIMH Project Accept, HPTN 043): a randomised study. Lancet Infect Dis 2011, 11:525-532.

56. Shott JP, Galiwango RM, Reynolds SJ: A quality management approach to Implementing point-of-care technologies for HIV diagnosis and monitoring in Sub-Saharan Africa. J Trop Med 2012, 2012:651927.

57. Delvaux T, Samreth S, Barr-DiChiara M, Seguy N, Guerra K, Ngauv B, Ouk V, Laga M, Mean CV: Linked response for prevention, care, and treatment of HIV/AIDS, STIs, and reproductive health issues: results after 18 months of implementation in five operational districts in Cambodia. $J$ Acquir Immune Defic Syndr 2011, 57:e47-e55.

58. Poudel KC, Fujita M, Green K, Poudel KT, Jimba M: Non-communicable diseases in South-East Asia. Lancet 2011, 377:2005-2005.

59. Rosen S, Fox MP: Retention in HIV Care between Testing and Treatment in Sub-Saharan Africa: A Systematic Review. PLoS Med 2011, 8:e1001056.

60. World Health Organization: Guidance on couples HIV testing and counseling including antiretroviral therapy for treatment and prevention in serodiscordant couples: Recommendations for a public health approach. Geneva: World Health Organization; 2012.

61. Jani IV, Sitoe NE, Alfai ER, Chongo PL, Quevedo Jl, Rocha BM, Lehe JD, Peter TF: Effect of point-of-care CD4 cell count tests on retention of patients and rates of antiretroviral therapy initiation in primary health clinics: an observational cohort study. Lancet 2011, 378:1572-1579.

62. Mtapuri-Zinyowera S, Chideme M, Mangwanya D, Mugurungi O, Gudukeya S, Hatzold K, Mangwiro A, Bhattacharya G, Lehe J, Peter T: Evaluation of the PIMA Point-of-Care CD4 Analyzer in VCT Clinics in Zimbabwe. J Acquir Immune Defic Syndr 2010, 55:1-7.
63. WHO Regional Office for the Western Pacific: People-Centered Health Care: A Policy Framework. Manila: World Health Organization Regional Office for the Western Pacific; 2007.

64. Joint United Nations Programme on HIV/AIDS (UNAIDS): Intensifying HIV Prevention: UNAIDS Policy Position Paper. Geneva: Joint United Nations Programme on HIV/AIDS; 2005.

doi:10.1186/1472-6963-12-483

Cite this article as: Fujita et al:: A new analytical framework of 'continuum of prevention and care' to maximize HIV case detection and retention in care in Vietnam. BMC Health Services Research 2012 12:483.

\section{Submit your next manuscript to BioMed Central and take full advantage of:}

- Convenient online submission

- Thorough peer review

- No space constraints or color figure charges

- Immediate publication on acceptance

- Inclusion in PubMed, CAS, Scopus and Google Scholar

- Research which is freely available for redistribution

Submit your manuscript at www.biomedcentral.com/submit
C) BioMed Central 